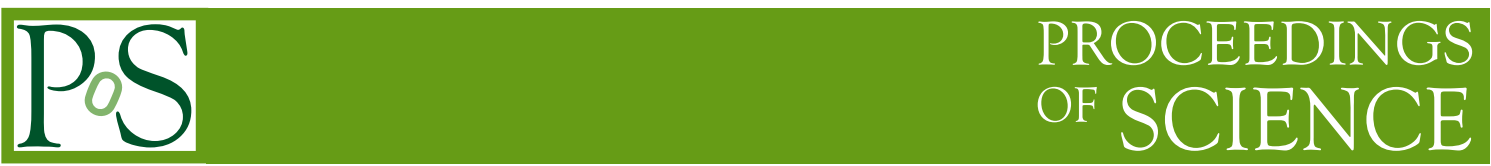

\title{
Quark Mass Variation and its effect on nuclear binding
}

\section{E. Carrillo-Serrano*}

CSSM and ARC Centre of Excellence for Particle Physics at the Tera-scale, School of Chemistry and Physics, University of Adelaide, Adelaide SA 5005, Australia

E-mail: manuel.carrilloserrano@adelaide.edu.au

\section{C. Cloët}

CSSM, School of Chemistry and Physics, University of Adelaide, Adelaide SA 5005, Australia E-mail: ian.cloet@adelaide.edu.au

\section{K. Tsushima}

CSSM, School of Chemistry and Physics, University of Adelaide, Adelaide SA 5005, Australia E-mail: kazuo.tsushima@adelaide.edu.au

\section{A. W. Thomas}

CSSM and ARC Centre of Excellence for Particle Physics at the Tera-scale,

School of Chemistry and Physics, University of Adelaide, Adelaide SA 5005, Australia

E-mail: anthony.thomaseadelaide.edu.au

\section{R. Afnan}

School of Chemical and Physical Sciences, Flinders University, GPO Box 2100, Adelaide SA 5001 Australia

E-mail: Iraj.Afnan@Flinders.edu.au

\begin{abstract}
We consider the variations of the nucleon mass and the masses of the mesons typically used in a one-boson exchange model arising from possible changes in light quark masses. These results are used to calculate the corresponding changes of the energy of the ${ }^{1} \mathrm{~S}_{0}$ anti-bound state and the binding energies of ${ }^{2} \mathrm{H},{ }^{3} \mathrm{H}$, and other nuclei. The possible consequences for the standard scenario for big bang nucleosynthesis are discussed [9]. In particular the possible solution of the ${ }^{7} \mathrm{Li}$ problem through a variation of quark masses is not supported.
\end{abstract}

XII International Symposium on Nuclei in the Cosmos,

August 5-12, 2012

Cairns, Australia

\footnotetext{
* Speaker.
} 


\section{INTRODUCTION}

Recently there has been growing evidence from quasar absorption spectra showing that the fine structure constant may have changed by an amount of around $10^{-5}$ in a billion years $[1,2,3,4]$. Although this evidence is still debatable [5], that variation can be related to higher changes in other fundamental quantities such as $\Lambda_{Q C D}$ and quark masses $[6,7,8]$. In particular a variation of the light quark masses $\delta m_{q} / m_{q}$ would be of order 38 times that of the fine structure constant $\delta \alpha / \alpha[6]$.

Different approaches to calculate the effect of these aparent quark mass changes in the nucleonnucleon force have been studied. These include: effective field theory [10,11], constraints from lattice QCD [12, 13], and calculations using the Argonne potential and the Schwinger-Dyson method [14].

In this work we calculate the variations with quark mass of the ${ }^{1} \mathrm{~S}_{0}$ anti-bound state, and the binding energies of ${ }^{2} \mathrm{H},{ }^{3} \mathrm{H}$, and other selected light nuclei [9]. This is studied considering the variations of the mass of each of the mesons that are employed in a one-boson exchange (OBE) picture of the nucleon-nucleon interaction.

These results are included into the quark-meson coupling model (QMC) for the calculations of the binding energies of ${ }^{7} \mathrm{Li},{ }^{12} \mathrm{C}$, and ${ }^{16} \mathrm{O}$ as well as using a typical OBE model for ${ }^{2} \mathrm{H}$, the ${ }^{1} \mathrm{~S}_{0}$ antibound state and ${ }^{3} \mathrm{H}[9]$.

Section II deals with the calculations for each of the mesons included and the nucleon. Sections III and IV give the results for the deuteron, triton and some other nuclei. Finally the possible effect to these results for big bang nucleosynthesis (BBN) is discussed [9], showing that in our model the ${ }^{7} \mathrm{Li}$ problem cannot be solved in this way.

\section{MESON AND NUCLEON MASSES}

We use three steps to calculate the changes in the meson masses with the variation of the current quark mass [9]. Firstly we used the Nambu-Jona-Laisino (NJL) model [16, 17] to calculate the relationship between the bare mass of the sigma meson $\left(m_{\sigma}^{(0)}\right)$ with the pion mass $\left(m_{\pi}\right)$, then we introduce the self energy contributions for the $\sigma, \rho$, and $\omega$ mesons. In the case of the $\sigma$ the inclusion was subjected to the position of the pole on the second sheet of the energy plane found by Leutwyler $e t$. al. [18]; for the $\rho$ and $\omega$ we use chiral fits from lattice QCD [22]. Finally all the results are translated to relationships with the quark mass through the Gell-Mann-Oakes-Renner (GMOR) relation. In the case of the nucleon mass, we use the recent calculations of the sigma commutator $\sigma_{\pi N}$ [23].

\subsection{VARIATION IN $\mathbf{m}_{\sigma}$ WITH $\mathbf{m}_{q}$}

With an OBE approach we parametrize the intermediate range attraction of the nucleonnucleon interaction through the exchange of a $\sigma$ meson [9]. Our results can be compared to [14] where a different approach is used. This should serve to pin down the uncertainties on this calculation. In our model the $\sigma$ can be composed of a "bare" part which coupled to two pions. The mass of the bare part is computed using the NJL model. For the rest of the contribution we calculate the propagator of the dressed $\sigma$, which can be described as an infinite sum of terms of the form of (a) in Fig. 1 (replacing $\rho$ by $\sigma$ ). Denoting the self-energy as $\Sigma_{\pi \pi}^{\sigma}$, the total propagator 
$\Delta_{\sigma}$ can be resumed to: $\Delta_{\sigma}=\frac{i}{p^{2}-\left(m_{\sigma}^{(0)}\right)^{2}+\Sigma_{\pi \pi}^{\sigma}}$. The pole of this propagator was calculated before by Leutwyler using the model independent method of the Roy equations [19], their result was: $p=m_{\sigma}-\frac{i}{2} \Gamma=441_{-8}^{+16}-i 272_{-12.5}^{+9} \mathrm{MeV}$, in agreement with the results given by the Particle Data Group (PDG) [20]. Our calculations should match this pole, and this allow us to fix $\Sigma_{\pi \pi}^{\sigma}$ in the form

$$
\sqrt{\left(m_{\sigma}^{(0)}\right)^{2}-\Sigma_{\pi \pi}^{\sigma}\left(m_{\sigma}^{2}\right)} \simeq 441-i 272 \mathrm{MeV}
$$

Respecting chiral symmetry, the expression for this self-energy is found with a derivative coupling between the bare $\sigma$ and the two pions

$$
i \Sigma_{\pi \pi}^{\sigma}=\frac{3}{2} \gamma_{0}^{2} \int \frac{d^{4} k}{(2 \pi)^{4}} \frac{\left[1-\frac{\left(\frac{p}{2}-k\right)^{2}}{\Lambda^{2}}\right]^{-4}\left[k^{\mu}(p-k)_{\mu}\right]^{2}}{\left(k^{2}-m_{\pi}^{2}\right)\left((p-k)^{2}-m_{\pi}^{2}\right)},
$$

with $k$ the momentum of the pion loop, $p$ the momentum of $\sigma, \gamma$ the coupling constant, and the first term in the numerator of the integrand is a dipole regulator with mass $\Lambda$. This integration is performed doing the temporal part first using contour integration, whereas the spatial component includes a rotation in the complex plane of $\vec{k}$ to ensure that the pole is in the second sheet. We then fit the three parameters: $\gamma, \lambda$, and $m_{\sigma}^{(0)}$ with the pole position [9].

Defining $m_{\sigma}^{2}(O B E)=\left(m_{\sigma}^{(0)}\right)^{2}-\Sigma_{\pi \pi}^{\sigma}(0)$ (i.e. at zero momentum transfer), any variation on $m_{\sigma}(O B E)$ with respect to $m_{\pi}$ is given by changes in $m_{\sigma}^{(0)}$ and $\Sigma_{\pi \pi}^{\sigma}(0)$. In addition, using the GellMann-Oakes-Renner (GMOR) relation [21], we finally can get the variation of $m_{\sigma}(O B E)$ with $\mathrm{m}_{q}$ by: $\frac{\delta m_{\sigma_{0(1)}}(O B E)}{m_{\sigma_{0(1)}}(O B E)}=0.089(0.072) \frac{\delta m_{q}}{m_{q}}$, with the indices 0 and 1 respectively correspond the scalar $\sigma_{0}$ and the second one to the isoscalar $\sigma_{1}$.

\subsection{VARIATIONS IN $\mathbf{m}_{\rho}$ AND $\mathbf{m}_{\omega}$ WITH RESPECT TO $\mathbf{m}_{q}$}

With the $\rho$ meson we follow the analysis done by Armour et. al. [22] using partially quenched lattice QCD data, and extrapolating to the physical limit for their fit. The self-energy diagrams relevant are shown in Fig. 1, where diagram (a) will be denoted as $\Sigma_{\pi \pi}^{\rho}$ and (b) as $\Sigma_{\pi \omega}^{\rho}$.

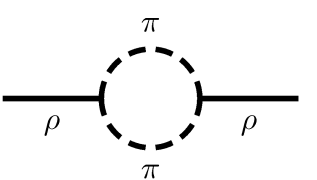

(a)

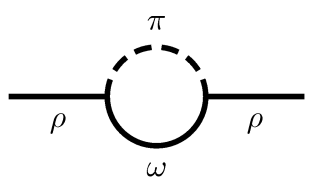

(b)

Figure 1: Self-energy contributions for the $\rho$-meson mass.

The expressions for these diagrams are regularized using dipole cutoffs in a similar way that was done for the $\sigma$ meson. The fit is then given by the following relation

$$
m_{\rho}=\sqrt{\left(a_{0}+a_{2} m_{\pi}^{2}+a_{4} m_{\pi}^{4}\right)^{2}+\Sigma_{T O T}},
$$


where $\Sigma_{T O T}=\Sigma_{\pi \pi}^{\rho}+\Sigma_{\pi \omega}^{\rho}$, and the coefficients, $a_{i}$, are: $a_{0}=832.00 \mathrm{MeV}, a_{2}=4.94 \times 10^{-4} \mathrm{MeV}^{-1}$, $a_{4}=-6.10 \times 10^{-11} \mathrm{MeV}^{-3}$ and $\Lambda=655.00 \mathrm{MeV}$ (up to errors). Simmilarly to the $\sigma$ case we consider almost zero momentum transfered in the OBE potential. We then can relate the changes in $m_{\rho}$ with $m_{\pi}$ according to the fit given in (2.3). The final variation gives: $\frac{\delta m_{\rho}}{m_{\rho}}=0.034 \frac{\delta m_{q}}{m_{q}}$.

In the case of the $\omega$ the procedure is basically the same, but with the difference that there is not an analogue of diagram (a) in Fig. 1, because it violates G-parity. In addition, since we have three ways to combine $\rho$ and $\pi$ : $\Sigma_{\rho \pi}^{\omega}=3 \times \Sigma_{\omega \pi}^{\rho}$. We use the same fit with the same coefficients as $\rho$, simply because their mass difference is around $10 \mathrm{MeV}$. For the coefficient for the variation in $m_{\omega}$, we obtain is: $\frac{\delta m_{\omega}}{m_{\omega}}=0.024 \frac{\delta m_{q}}{m_{q}}[9]$.

\subsection{NUCLEON MASS}

For the nucleon mass we invoque the $\pi N$ sigma commutator

$$
\sigma_{\pi N}=m_{q}\langle N|\bar{q} q| N\rangle=m_{q} \frac{\delta m_{N}}{\delta m_{q}}
$$

where $\bar{q} q \equiv \bar{u} u+\bar{d} d$. This result comes from the Feynman-Hellmann theorem. To extract this value we use the analysis done by Shanahan et. al. [23] of the PACS-CS data, $\sigma_{\pi N}=45 \pm 6 \mathrm{MeV}$. This finally gives a relation: $\frac{\delta m_{N}}{m_{N}}=0.048 \frac{\delta m_{q}}{m_{q}}$.

\section{3. ${ }^{7} \mathrm{Li},{ }^{12} \mathrm{C}$ AND ${ }^{16} \mathrm{O}$ NUCLEI}

To calculate the single-particle energies of ${ }^{7} \mathrm{Li},{ }^{12} \mathrm{C}$ and ${ }^{16} \mathrm{O}$ nuclei we use the quark-meson coupling model, a nuclear model based on quark degrees of freedom [24]. We then calculate the changes in these binding energies with variations in the current quark mass $m_{q}$ [9].

We took standard values for mesons and nucleon masses as well as the $\sigma-N$ coupling constant and the rms charge radius of ${ }^{40} \mathrm{Ca}$ [25].

The final results for binding energies per nucleon as a function of the quark mass are:

$$
\begin{aligned}
& \frac{\delta\left|\mathrm{E}_{7 \mathrm{Li}}\right| / \text { nucleon }}{\left|\mathrm{E}_{7 \mathrm{Li}}\right| / \text { nucleon }}=-2.571 \frac{\delta m_{q}}{m_{q}}, \\
& \frac{\delta\left|\mathrm{E}_{{ }^{12} \mathrm{C}}\right| / \text { nucleon }}{\left|\mathrm{E}_{{ }^{12} \mathrm{C}}\right| / \text { nucleon }}=-1.438 \frac{\delta m_{q}}{m_{q}}, \\
& \frac{\delta\left|\mathrm{E}_{{ }^{16} \mathrm{O}}\right| / \text { nucleon }}{\left|\mathrm{E}_{16 \mathrm{O}}\right| / \text { nucleon }}=-1.082 \frac{\delta m_{q}}{m_{q}} .
\end{aligned}
$$

\section{VARIATION IN THE ENERGIES OF THE TWO- AND THREE-NUCLEON SYSTEMS WITH VARIATION IN THE MESON AND NUCLEON MASSES}

For the deuteron, the antibound ${ }^{1} \mathrm{~S}_{0}$ state and the triton; we consider an OBE potential. We choose the Bryan-Scott (BS) approach because it employs the mesons we have considered $\left(\left(\pi, \eta, \sigma_{0}, \sigma_{1}, \rho, \omega\right)[26]\right.$. For the anti-bound state ${ }^{1} \mathrm{~S}_{0}$, the location of the pole is in the second energy sheet, which makes the evaluation more complicated in order to apply the method of moments. This problem is overcome by approximating to the zero energy point, because its energy is 
close to zero $\left(\mathrm{E}_{p}=-0.066 \mathrm{MeV}\right)$. The next step is to include a separable potential that allows us to write the Fadeev equations as a set of coupled one dimensional integral equations.

Varying the values of the masses of the mesons included permits us to find the changes in the energies of the deuteron, the ${ }^{1} \mathrm{~S}_{0}$ state and the triton. Finally, using the GMOR relation, and the coefficients relating the meson masses and the quark mass, we get the following expressions [9]:

$$
\begin{aligned}
& \frac{\delta E_{D}}{E_{D}}=-0.912 \frac{\delta m_{q}}{m_{q}}, \\
& \frac{\delta E_{t}}{E_{t}}=-0.980 \frac{\delta m_{q}}{m_{q}}, \\
& \frac{\delta E_{P}}{E_{P}}=-2.839 \frac{\delta m_{q}}{m_{q}} .
\end{aligned}
$$

\section{CONCLUSION}

Defining the parameter $K_{A}$ as

$$
\frac{\delta B E(A)}{B E(A)}=K_{A} \frac{\delta m_{q}}{m_{q}}
$$

our results for $K_{D}$ and $K_{t}$ are in agreement with [14]. However, for the ${ }^{1} \mathrm{~S}_{0}$ state the disagreement is considerable, since the signs are different (in our case $K_{P}$ is negative whereas using the relationship with $E_{D}$ in [15] it is positive).

For the light nuclei the values of $K_{A}$ deduced for ${ }^{12} \mathrm{C}$ and ${ }^{16} \mathrm{O}$ agree with those found in [14]. However it is noticeable that for ${ }^{7} \mathrm{Li}$ our value is larger than the value they reported. These authors claim that the uncertainty can be as large as a factor of two, and our value is within this range.

Our motivation for the study of these variations with quark mass, comes from the possible effects on BBN. In particular, the discrepancy of the abundance of ${ }^{7} \mathrm{Li}$ with the present baryon to photon ratio, can be linked to the variation of the deuteron binding energy with the quark mass [15]. Our results are summarized in Fig. 2 where the curves correspond to the values of $K_{D}$ and $K_{P}$ calculated here (solid line), as well as the values used by Berengut et. al. [27]. In that reference the range of values for $\frac{\delta m_{q}}{m_{q}}$ are chosen such that the Lithium problem can be solved. However, even within that range our results show that such variations in the quark mass (at least for the $p(n, \gamma) d$ reaction) does not yield a significant change in the ${ }^{7} \mathrm{Li}$ abundance. The reason is that the contribution from the deuteron tends to cancel the one from the ${ }^{1} \mathrm{~S}_{0}$ state [9]. It is different from [15] because their sign for the ${ }^{1} S_{0}$ is opposite to ours. Therefore their contributions are enhanced. We finally note that it will be necessary to include the variation of the strange quark mass in the calculations, especially with the new findings for the sigma commutators.

\section{AKNOWLEDGEMENTS}

This work was supported by the Australian Research Council through the ARC Centre for Excellence in Particle Physics at the Terascale and through the Australian Laureate Fellowship (FL0992247), as well as by the University of Adelaide. 


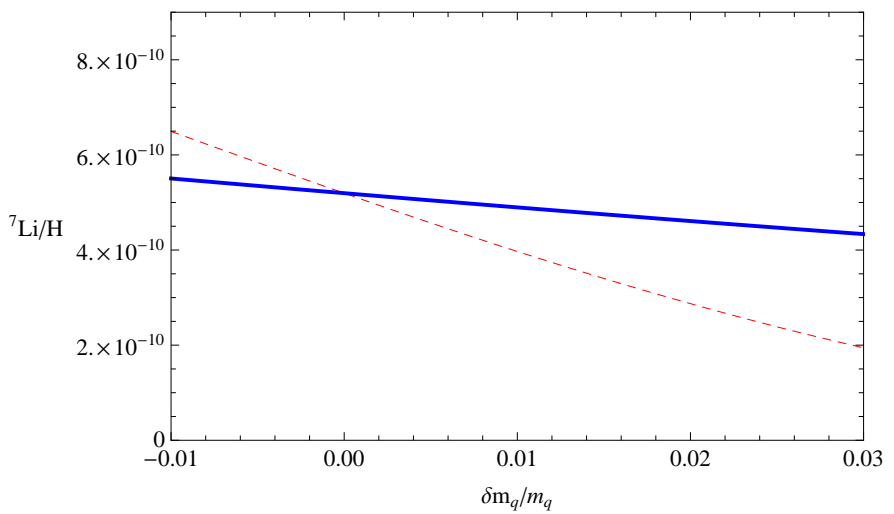

Figure 2: (Color online) Abundance of ${ }^{7} \mathrm{Li}$ with respect to changes in the quark mass in $p(n, \gamma) d$ calculated in the same way as [27] (dashed-red line) and using our results for $K_{D}$ and $K_{P}$ (continuous-blue line).

\section{References}

[1] V. A. Dzuba, V. V. Flambaum and J. K. Webb, Phys. Rev. [15] V. F. Dmitriev, V. V. Flambaum and J. K. Webb, Phys. Rev. Lett. 82, 888 (1999). D 69, 063506 (2004).

[2] J. K. Webb et al., Phys. Rev. Lett. 82, 884 (1999).

[16] Y. Nambu and G. Jona-Lasinio, Phys. Rev. 122, 345 (1961).

[3] M. T. Murphy, J. K. Webb and V. V. Flambaum, Mon. Not. [17] W. Bentz and A. W. Thomas, Nucl. Phys. A 696, 138 (2001). Roy. Astron. Soc. 345, 609 (2003).

[4] H. B. Sandvik, J. D. Barrow and J. Magueijo, Phys. Rev. Lett. 88, 031302 (2002).

[18] I. Caprini, G. Colangelo, H. Leutwyler, Phys. Lett. 96, 132001 (2006).

[19] B. Ananthanarayan, G. Colangelo, J. Gasser, H. Leutwyler, Phys. Rep. 353, 207-297 (2001).

[5] R. Srianand, H. Chand, P. Petitjean and B. Aracil, Phy. Rev. Lett. 92, 121302 (2004).

[6] W. J. Marciano, Phys. Rev. Lett. 52, 489 (1984). X. Calmet, H. Fritzsch, Eur. Phys. J. C24, 639 (2002).

P. Langacker, G . Segré, M. J. Strassler, Phys. Lett. B528, 121-128 (2002).

[7] J. D. Bekenstein, Phys. Rev. D 25, 1527 (1982). S. M. Roy, Phys. Lett. 36B, 353 (1971).

[20] Particle Data Group, Phys. Lett. D66, 1 (2002).

[21] P. Gell-Mann, R. J. Oakes, B. Renner, Phys. Rev. 175, 2195 (1968).

[22] W. Armour, C. R. Allton, D. .B. Leinweber, A. W. Thomas, R. D. Young, J. Phys. G. 32, 971 (2006);

D. B. Leinweber, A. W. Thomas, K. Tsushima, S. V. Wright, Phys. Rev. D64, 094502 (2001);

C. R. Allton, W. Armour, A. W. Thomas, R. D. Young, Phys. Lett. B628, 125-130 (2005).

[9] M. E. Carrillo-Serrano, I. C. Clöet, K. Tsushima, A. W. Thomas and I. R. Afnan, Phys. Rev. C 87, 015801 (2013).

[10] S. R. Beane and M. J. Savage, Nucl. Phys. A 713, 148 (2003).

[11] E. Epelbaum, U. -G. Meissner and W. Gloeckle, Nucl. Phys A 714, 535 (2003).

[12] S. R. Beane and M. J. Savage, Nucl. Phys. A 717, 91 (2003)

[13] J. Soto and J. Tarrus, Phys. Rev. C 85, 044001 (2012)

[14] V. V. Flambaum, R. B. Wiringa, Phys. Rev. C76, 054002 (2007).

[23] P. E. Shanahan, A. W. Thomas and R. D. Young, arXiv:1205.5365 [nucl-th].

[24] P. A. M. Guichon, Phys. Lett. B200, 235 (1988); P. A. M. Guichon, K. Saito, E. N. Rodionov, A. W. Thomas, Nucl. Phys. A601, 349 (1996).

[25] K. Saito, K. Tsushima, A. W. Thomas, Nucl. Phys. A609, 339 (1996).

[26] Roland Bryan, and Bruce L. Scott, Phys. Rev. 177, 1435 (1969).

[27] J. C. Berengut, V. V. Flambaum and V. F. Dmitriev, Phys. Lett. B 683, 114 (2010). 